\title{
COUPLING OF THE FLUX DIFFUSION EQUATION WITH THE EQUILIBRIUM RECONSTRUCTION AT
} ASDEX UPGRADE

\author{
R. Fischer ${ }^{1}$, A. Bock ${ }^{1}$, M. Dunne ${ }^{1}$, J.C. Fuchs ${ }^{1}$, L. Giannone ${ }^{1}$, K.

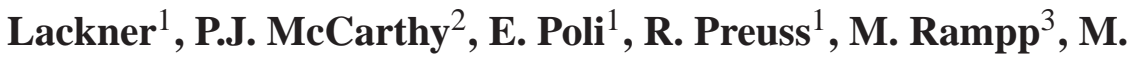

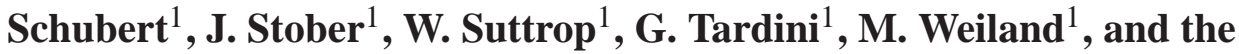 \\ ASDEX Upgrade Team ${ }^{1}$ \\ ${ }^{1}$ Max-Planck-Institut für Plasmaphysik, Boltzmannstr. 2, D-85748 Garching, Germany \\ Rainer.Fischer@ipp.mpg.de \\ ${ }^{2}$ Department of Physics, University College Cork, Cork, Ireland. \\ ${ }^{3}$ Max Planck Computing and Data Facility, Gießenbachstr. 2, 85748 Garching, Germany
}

\begin{abstract}
Tokamak equilibrium reconstruction can benefit much from internal measurements of the current distribution. In lacking robust internal measurements the reconstruction is ill-posed in the plasma core, not allowing for a sensible estimation of the current distribution. Such deficiencies can be compensated by modelling of the current distribution evolution by employing the current diffusion equation between successive equilibria. A scheme for the coupling of the predictive current diffusion equation with the equilibrium reconstruction from an inverse Grad-Shafranov equilibrium solver minimising a least-squares criterion on measured and modelled data is proposed. The scheme is intended for routine equilibrium analysis shortly after the discharge where all diagnostic data are available. Results from the implementation at ASDEX Upgrade are shown, applied to a reversed-shear plasma with counter-current ECCD and to the start-up phase of the plasma. Results are compared to TRANSP calculations.
\end{abstract}




\section{INTRODUCTION}

The magnetic confinement of a tokamak plasma is routinely calculated by solving the GradShafranov equation (GSE) describing the ideal magneto-hydrodynamic (MHD) equilibrium for the poloidal flux function $\psi$ for axisymmetric geometry. For advanced operation of fusion devices a reasonable knowledge of the current distribution and, hence, the $q$-profile is mandatory. Routine offline as well as real-time reconstruction of the equilibrium mainly relies on magnetic measurements, as high-quality internal measurements of the current distribution are not always present. Motional stark effect (MSE) data and polarimetry measurements can provide internal information about the current distribution but are often cumbersome to calibrate. Furthermore, the set of MSE data available is often not capable of resolving the ill-posedness of the equilibrium reconstruction completely and polarimetry measurements provide line-integral information only. The lack of robust current measurements results in an ill-posed equilibrium reconstruction problem in the plasma core which can be tackled either by a reduced set of basis functions (parameters) or by regularization applying spatial smoothness constraints to the source profiles of the GSE. In both cases, the resulting current distribution in the plasma core does not reflect any measured information about the current distribution but sensitively depends on the non-physical regularization condition. Additionally, without temporal smoothing the current distributions of successive equilibria often show unreasonable variability not consistent with the current diffusion time.

The ill-posedness of the equilibrium reconstruction due to the lack of a sufficient set of measurements can be reduced by augmenting the set of measurements by plasma modelling results. To improve the equilibrium without relying much on smoothness constraints, the present work proposes a coupling scheme of a GSE solver with the results of the current diffusion equation (CDE). As the $\mathrm{CDE}$ predictively models the evolution of current profiles to be used for the next equilibrium reconstruction, the inverse GSE solver minimises a least- 
COUPLING OF THE FLUX DIFFUSION EQUATION WITH THE EQUILIBRIUM RECONSTRUCTION AT ASD squares criterion on measured and modelled data.

The deployment of the CDE can be found in various equilibrium and plasma modelling codes but is, to our knowledge, not used so far in any real-time or offline equilibrium reconstruction in the proposed way. The popular transport and equilibrium code ASTRA ${ }^{1,2}$ provides a well established numerical tool for the analysis and modelling of magnetically confined plasmas. A recent variant of ASTRA $^{3}$ shows a similar coupling of the GSE and the CDE, although an iteration process of the two solvers of GSE and CDE has to ensure consistency between the two solutions. Typically in such coupling schemes the original GS equilibrium boundary is used as a fixed-boundary constraint, and the internal flux distribution is reconstructed in a second step. In these weak coupling schemes the codes run sequentially. The coupling of the GSE and CDE in the present work does not have to rely on such iterations, since the boundary of the equilibrium is determined robustly from magnetic measurements, measurements of the current in external coils, measurements of the divertor tile currents, and the pressure profiles available from kinetic profile diagnostics at each time point an equilibrium is calculated (typically every $1 \mathrm{~ms}$ ). Due to its high level of sophistication and due to its main purpose of plasma modelling, ASTRA was not applied so far as a data-interpretative equilibrium solver at ASDEX Upgrade. The highlydeveloped tokamak transport and data analysis code TRANSP ${ }^{4}$ is used for dedicated analysis only, due to its rather large computational resources. As with ASTRA, the high level of sophistication of TRANSP prevented, so far, a routine analysis of the equilibrium shortly after a plasma discharge. Nevertheless, TRANSP provides, amongst other quantities, an improved equilibrium compared to classical GSE solvers which makes it an ideal candidate for benchmarking of the present results. The RAPTOR code ${ }^{5}$ solves flux-surface averaged 1.5 D equations for particle and heat transport and includes the CDE for magnetic field resistive diffusion. RAPTOR coupled with the LIUQE ${ }^{6}$ equilibrium solver is designed for real-time 
COUPLING OF THE FLUX DIFFUSION EQUATION WITH THE EQUILIBRIUM RECONSTRUCTION AT ASD analysis and prediction of the plasma employing appropriate approximations necessary for real-time capability. Up to now the RAPTOR code is not providing at ASDEX Upgrade the current distribution for the subsequent real-time equilibrium evaluation ${ }^{7}$ as proposed in the present work for offline analysis, although the idea first emerged in ${ }^{5}$ in the context of real-time simulations on ITER.

The coupling of the GSE and CDE solvers is performed within the equilibrium package IDE (Integrated Data analysis Equilibrium) ${ }^{8}$ which is a free-boundary equilibrium code used at ASDEX Upgrade. The IDE package supplements the standard CLISTE equilibrium reconstruction ${ }^{9}$ as it allows within a flexible and modular code environment straightforward combination of a set of measurements for magnetic reconstruction with measurements of kinetic profiles from the Integrated Data Analysis (IDA) suite ${ }^{10}$. Furthermore, it can easily be extended with heterogeneous other measurements and physics modelling as presented in this work. Another advantage of the present method compared to ASTRA and TRANSP is that it provides an interpretative free-boundary equilibrium where magnetic and other measured data (see below) are fitted. Additionally, the code is real-time capable in both parts, the GSE solver ${ }^{11,12,13}$ and the current diffusion solver ${ }^{5}$, which is far beyond the scope of codes such as TRANSP and ASTRA. The primary goal is to provide equilibria with a temporal resolution of $1 \mathrm{~ms}$ for the full ASDEX Upgrade discharge of up to $10 \mathrm{~s}$ before the next discharge is launched typically after about $30 \mathrm{~min}$. Within the IDA approach the equilibrium using kinetic profiles is evaluated consistently with the kinetic profiles which themselves depend on the equilibrium. Since the present work is dedicated for routine analysis shortly after the discharge where all diagnostic data are available, the IDE package should be interpreted as a link between the routinely used equilibrium codes running in offline mode without modelling support and the highly sophisticated codes for plasma analysis and prediction.

The coupling of the GSE and CDE solvers is applied to two examples from the ASDEX 
Upgrade tokamak: A counter-current experiment driven by electron cyclotron current drive (ECCD) leading to the development of a reversed-shear plasma, and the start-up phase of the plasma. Results are compared to TRANSP calculations.

Section II introduces the equilibrium package IDE solving the GSE, the CDE, and the coupling of both. Section III shows results and section IV concludes and gives an outlook.

\section{EQUILIBRIUM EQUATIONS}

This chapter summarises the Grad-Shafranov solver implemented in the equilibrium package IDE, the current diffusion solver, and describes in detail the coupling of the two.

\section{II.A. Grad-Shafranov Solver}

The GS equation describing ideal magneto-hydrodynamic equilibrium in two-dimensional tokamak geometry reads

$$
\left(R \frac{\partial}{\partial R} \frac{1}{R} \frac{\partial}{\partial R}+\frac{\partial^{2}}{\partial z^{2}}\right) \psi=-2 \pi \mu_{0} R j_{\phi}(\psi),
$$

where $\psi(R, z)$ denotes the poloidal flux function in cylindrical coordinates $(R, z)$. The toroidal current density profile, $j_{\phi}$, consists of two terms,

$$
j_{\phi}:=2 \pi\left(R \frac{\partial p(\psi)}{\partial \psi}+\frac{F(\psi)}{\mu_{0} R} \frac{\mathrm{d} F(\psi)}{\mathrm{d} \psi}\right),
$$

where $p(\psi)$ is the plasma pressure (isotropic case) and $F(\psi)=\mu_{0} I_{\mathrm{pol}} /(2 \pi)$ is proportional to the total poloidal current $I_{\mathrm{pol}}$.

The inertia due to toroidal plasma rotation can be considered by an extension of the GSE if the centrifugal force is expected to be large enough ${ }^{14}$. Since it was shown that inertia has to be considered only for toroidal Mach numbers larger than 0.2 , it is not considered to be relevant for the data presented in this work.

Since 2014 ASDEX Upgrade is equipped with toroidally symmetric rows of ferromagnetic tiles ${ }^{15}$. The effect of the ferromagnetic rows on the equilibrium is small but, 
COUPLING OF THE FLUX DIFFUSION EQUATION WITH THE EQUILIBRIUM RECONSTRUCTION AT ASD nevertheless, included in the equilibrium solver. Details can be found in ${ }^{7}$.

Eq. (1) is solved with a parallel Grad-Shafranov solver capable for real-time control of tokamak plasmas ${ }^{11,12,13}$. The solver is embedded in the equilibrium package IDE (Integrated Data analysis Equilibrium $)^{8}$. The IDE code can be run in an interpretative mode of reconstructing an equilibrium from minimising a least-squares criterion on measured data analogous to the concept of CLISTE$^{9}$ as well as in a predictive mode for given source profiles ${ }^{14}$. This work relies on the interpretative mode fitting various measured data sets and plasma modelling results as shown below. The modular IDE package is written in FORTRAN90 and provides a flexible framework to be easily extended with additional measurement or modelling constraints. The present measurement constraints implemented are given by magnetic measurements from poloidal and radial field coils and flux loops, motional stark effect (MSE), polarimetry (Faraday rotation), pressure constraints, divertor tile currents, loop voltage, iso-flux constraints ${ }^{8}$, q-value constraints, currents in external poloidal field coils, and flux-surface averaged toroidal current density which is subject of the present work. All measurements are within the poloidal field grid such that the poloidal flux outside the grid is not needed. Additional (non-physical) smoothness constraints are applied to the source profiles $p^{\prime}$ and $F F^{\prime}$ since the number of basis functions is chosen sufficiently large to allow for equilibria sufficiently flexible to address all occurring plasma scenarios 9 .

The pressure constraint consists of the sum of the electron and ion thermal pressure and the pressure of the fast ions. The electron thermal pressure profile is provided by the IDA suite ${ }^{10}$. The ion thermal pressure is calculated from the ion temperature taken from charge exchange measurements, if available, or $T_{\mathrm{i}}=T_{\mathrm{e}}$ is assumed, and the ion density using the electron density (IDA) and a $Z_{\text {eff }}$ profile ${ }^{16}$. The pressure of the fast ions are taken, for the moment, from TRANSP calculations. To replace the time consuming TRANSP runs, a reasonable approximation of the fast-ion pressure profile is currently under development. The 
COUPLING OF THE FLUX DIFFUSION EQUATION WITH THE EQUILIBRIUM RECONSTRUCTION AT ASD pressure constraints at the plasma edge ${ }^{17}$ as well as in the plasma core allows to reduce the illposedness of the $p^{\prime}$-term of the equilibrium reconstruction. The pressure profile establishes the bootstrap current profile ${ }^{17}$, but it is not able to constrain the current distribution in the plasma core significantly.

The standard analysis is performed using magnetic measurements, measurements of the divertor tile currents to allow for constraints of the current in the scrape-of-layer (SOL), pressure constraints in the region around the last-closed-flux-surface from the IDA analysis ${ }^{10}$ considering thermal pressure only, and currents in external poloidal field coils. These data sets are routinely available. Close to the separatrix no significant fast-ion pressure is expected. Some flexibility in the poloidal field coil currents is allowed to compensate for induced vessel currents mainly relevant in the ramp-up and ramp-down phases. The other data sets and constraints $(\mathrm{MSE}, \ldots)$ are applied as available.

A standard set of output quantities is written into the ASDEX Upgrade shotfile system to be compatible with all the existing access and analysis tools. An additional set of signals and profiles defining all facets of the equilibrium is stored including their uncertainties. Furthermore, the measured and modelled data and constraints are stored augmented with the residues to allow for detailed validation of the reconstructed equilibrium retrospectively.

\section{II.B. Current Diffusion}

The CDE (also known as poloidal flux diffusion equation) can be written as ${ }^{1,5}$

$$
\sigma_{\|} \frac{\partial \psi}{\partial t}=\frac{R_{0} J^{2}}{\mu_{0} \rho} \frac{\partial}{\partial \rho}\left(\frac{G_{2}}{J} \frac{\partial \psi}{\partial \rho}\right)-\frac{V^{\prime}}{2 \pi \rho}\left(j_{\mathrm{bs}}+j_{\mathrm{cd}}\right)
$$

assuming $\partial B_{0} / \partial t=0 . \psi(\rho)$ is the poloidal magnetic flux at

$$
\rho \equiv \rho_{\text {tor }}:=\sqrt{\frac{\Phi}{\pi B_{0}}}
$$

with the toroidal magnetic flux $\Phi$ and the vacuum magnetic field $B_{0}=B\left(R_{0}\right)$ at the reference radius $R_{0}$. The CDE describes, therefore, the diffusion of the poloidal flux on the background 
of the toroidal flux due to resistivity. The quantities $J, V^{\prime}$ and $G_{2}$ are defined as:

$$
\begin{aligned}
J & :=1-\frac{\mu_{0}}{2 \pi R_{0} B_{0}} \int_{S_{\theta}} \mathbf{j} \cdot d \mathbf{S}_{\theta} \\
V^{\prime} & :=\frac{\partial V}{\partial \rho} \\
G_{2} & :=\frac{V^{\prime}}{4 \pi^{2}}\left\langle\left(\frac{\nabla \rho}{R}\right)^{2}\right\rangle
\end{aligned}
$$

where the flux-surface average of an arbitrary function $f$ is given by ${ }^{1}$ :

$$
\langle f\rangle=\oint f \frac{d l_{\theta}}{B_{\mathrm{pol}}} / \oint \frac{d l_{\theta}}{B_{\mathrm{pol}}}
$$

Various flux-surface averaged quantities are calculated from contour integrations on the poloidal flux grid for a given equilibrium. Since there is only a reduced number of contour points available close to the plasma center, the flux-surface averaged quantities have to be properly extrapolated from a region with a sufficient large number of contour points to the region close to the plasma center. For this purpose, an additional useful relation is given by:

$$
G_{3}:=\left\langle\frac{R_{0}^{2}}{R^{2}}\right\rangle \equiv \frac{4 \pi^{2} \rho R_{0}}{J V^{\prime}}
$$

For a given equilibrium, $G_{3}$ can easily be calculated from contour integrations on the poloidal flux grid. The extrapolation of $G_{3}$ for $\rho_{\text {pol }}<0.12$ is performed linearly in $\rho_{\text {pol }}$ to $G_{3}\left(\rho_{\text {pol }}=0\right)=R_{0}^{2} / R_{\text {mag }}^{2}$. The complete $G_{3}$-profile allows a straightforward evaluation of $V^{\prime}$ for a given equilibrium.

From the parameterisation of the given equilibrium the $J$-profile and the profile of the flux-surface averaged toroidal current density $j_{\text {tor }}$ can easily be calculated. According to Eq. (2) $j_{\text {tor }}$ is given by

$$
j_{\text {tor }}(\rho)=2 \pi\left(\langle R\rangle p^{\prime}+\left\langle\frac{1}{\mu_{0} R}\right\rangle F F^{\prime}\right)
$$

where $p^{\prime}$ and $F F^{\prime}$ are calculated from the weighted sum of basis functions known from the previous equilibrium reconstruction. $\langle R\rangle$ and $\langle 1 / R\rangle$ are evaluated from contour integrations with a quadratic interpolation to the radial position of the magnetic axis $R_{\text {mag. }}$. 
Numerical integration of $j_{\text {tor }}$ using ${ }^{1}$

$$
I_{\mathrm{pl}}(\rho)=\frac{1}{2 \pi R_{0}} \int_{0}^{\rho} V^{\prime} j_{\text {tor }} d \hat{\rho}
$$

provides the plasma current $I_{\mathrm{pl}}$ inside a $\rho$ surface. Employing

$$
\begin{aligned}
I_{\mathrm{pl}} & =\frac{G_{2}}{\mu_{0}} \frac{\partial \psi}{\partial \rho} \\
\frac{\partial \psi}{\partial \rho} & =\frac{2 \pi B_{0} \rho}{q}
\end{aligned}
$$

one obtains

$$
G_{2}=\frac{\mu_{0} I_{\mathrm{p} 1} q}{2 \pi B_{0} \rho}
$$

which can readily be obtained for a given equilibrium without needing an extrapolation to the plasma center. In contrast, Eq. (7) is based on contour integrations which is less precise close to the plasma center.

The parallel conductivity $\sigma_{\|}:=\sigma_{\text {neo }}=c_{\text {neo }} \sigma_{\text {spitz }}$ decomposes into the Spitzer conductivity $\sigma_{\text {spitz }}$ and a neoclassical correction term $c_{\text {neo }}$ considering trapped particles ${ }^{18,5}$. The fraction of trapped electrons is approximated with $f_{\mathrm{tr}}=\sqrt{1-R_{\min } / R_{\max }} 19$ where $R_{\min }\left(R_{\max }\right)$ is the minimum (maximum) major radius of the corresponding flux surface, respectively. The minor radius is defined by $r=\left(R_{\max }-R_{\min }\right) / 2$. For calculating the collisionality $v_{\mathrm{e} *}$, the electron density and temperature calculated with the IDA ${ }^{10}$ approach is used. The equilibrium needed in the IDA approach for mapping of the various diagnostics on a common magnetic coordinate system can be calculated self-consistently by iterating the equilibrium and the profile reconstruction.

The bootstrap current $j_{\mathrm{bs}}$ is calculated as described in detail in ${ }^{18,20,5}$.

The auxiliary current drive $j_{\mathrm{cd}}=j_{\mathrm{ec}}+j_{\mathrm{nb}}$ at ASDEX Upgrade is given by electron cyclotron current drive (ECCD) $j_{\mathrm{ec}}$ and neutral beam current drive (NBCD) $j_{\mathrm{nb}}$. The ECCD is calculated using the TORBEAM code ${ }^{21}$ where up to 8 gyrotron launchers are available. The NBCD is taken, for the moment, from TRANSP calculations. As for the pressure profile of the 

1 shows current densities from NBCD, ECCD with 4 gyrotrons, and the bootstrap current. In this example, the gyrotrons were positioned such that a large counter-current close to the

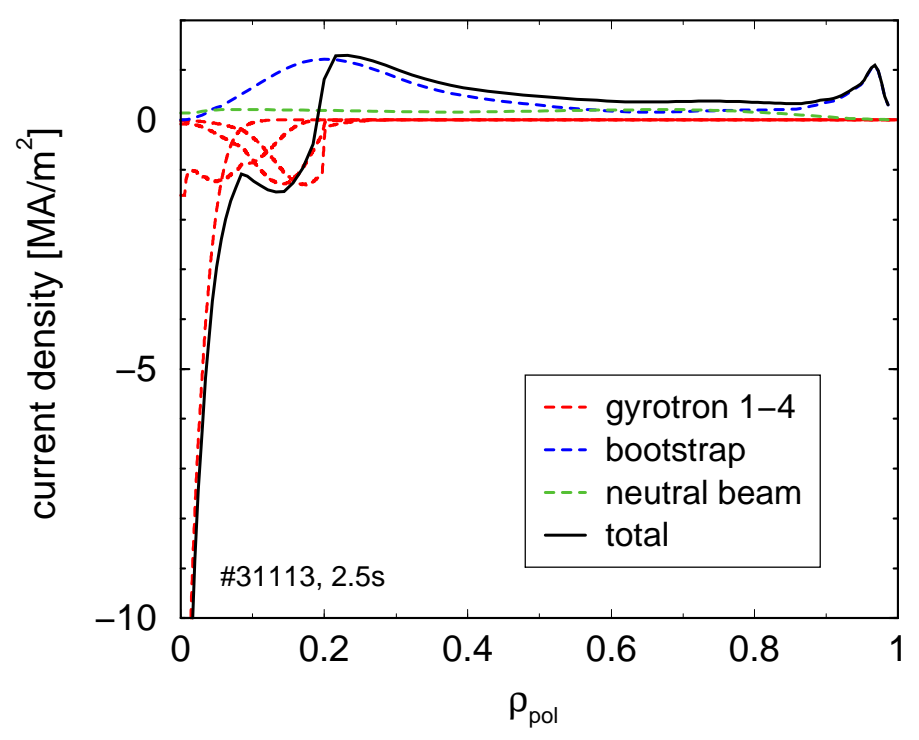

Fig. 1. Driven current from NBCD and ECCD with 4 gyrotrons, and the bootstrap current for $\# 31113$ at $2.5 \mathrm{~s}$.

plasma center is produced.

The boundary term needed for the CDE is specified by the plasma current $I_{\mathrm{p}}$, which is known sufficiently well from the magnetic measurements:

$$
\left.\frac{\partial \psi}{\partial \rho}\right|_{\rho_{\mathrm{e}}}=\frac{\mu_{0}}{G_{2}\left(\rho_{\mathrm{e}}\right)} I_{\mathrm{p}}(t)
$$

For a given set of the profiles $J$ and $\sigma_{\|}$, and of the metric quantities $G_{2}, G_{3}, V^{\prime}$ the CDE is integrated in time. The CDE poses an initial value problem of a partial differential equation (PDE) which is solved with a Crank-Nicholson discretization with an average of an explicit and implicit scheme ${ }^{22}$ applied (1) to the poloidal flux, and (2) to the coefficients of the finite elements of the poloidal flux as described in detail in ${ }^{5}$. Both methods were implemented and the results were compared. They produce very similar results validating the correct implementation of the PDE solvers. 


\section{II.C. Coupling of Equilibrium Solver with Current Diffusion}

The coupling of the GSE solver with the CDE solver is performed successively in time. One time step consists of two parts. The first part is given by a numerical solution of the CDE Eq. (3) initialised with an equilibrium given at time $t$. The CDE is integrated until time $t+\Delta t$, where the next equilibrium is to be calculated. The second part is given by solving the GSE Eq. (1) at time $t+\Delta t$ with additional constraints from the results of the CDE solver. The additional constraints are given by the toroidal current distribution averaged on various poloidal flux surfaces. A scheme of the workflow is depicted in Figure 2.

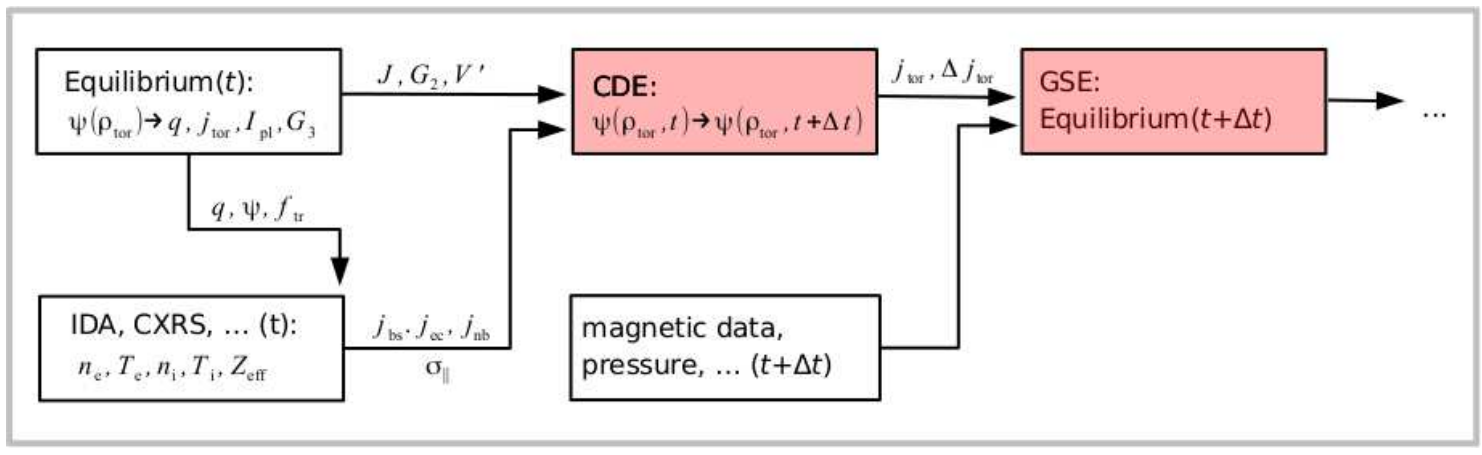

Fig. 2. Diagram illustrating the workflow of the coupling of the GSE solver with the CDE solver.

The details of the two solver steps are as follows: From the previous time step or from an initial equilibrium at time $t$ the $\mathrm{CDE}$ solver is initialised. An equidistant $\rho\left(\equiv \rho_{\text {tor }}\right)$ grid is defined between the magnetic axis and the last closed flux surface. On this grid Eq. (3) will be solved numerically. The corresponding poloidal flux $\psi(\rho)$ on the grid is calculated by integrating $q=d \Phi / d \psi$ starting at the magnetic axis. The $q$-profile is given by the equilibrium at time $t$. The parameterisation of the previous equilibrium allows to calculate the $J$-profile. $G_{3}$ and $V^{\prime}$ are evaluated as a function of $\rho$ from integration along contour lines of the poloidal flux grid. The contour lines also provide $R_{\min }, R_{\max }$ and $f_{\text {tr }}$. From the averaged toroidal current density $j_{\text {tor }}$ (Eq. (10)) the plasma current $I_{\mathrm{pl}}(\rho)$ inside a $\rho$ surface is evaluated which allows to 
COUPLING OF THE FLUX DIFFUSION EQUATION WITH THE EQUILIBRIUM RECONSTRUCTION AT ASD

calculate $G_{2}(\rho)$. The parallel conductivity $\sigma_{\|}(\rho)$ and the bootstrap current are calculated with temperature and density profiles from various profile diagnostics. The electron temperature $T_{\mathrm{e}}$ and density $n_{\mathrm{e}}$ profiles are taken from an IDA analysis of, typically, electron cyclotron emission, lithium beam and interferometry diagnostics ${ }^{10}$. The ion temperature $T_{\mathrm{i}}$ is taken from charge exchange measurements, if available, or $T_{\mathrm{i}}=T_{\mathrm{e}}$ is assumed. The ion density $n_{\mathrm{i}}$ is calculated from $n_{\mathrm{e}}$ and a $Z_{\mathrm{eff}}$ profile ${ }^{16}$. From the electron profiles and the (time varying) setting of the ECRH system the electron cyclotron driven current density $j_{\mathrm{ec}}$ for each gyrotron launcher is calculated employing the TORBEAM code. The neutral beam current drive $j_{\mathrm{nb}}$ is taken momentarily from TRANSP calculations. This concludes the initialisation of the CDE solver.

The CDE solver is iterated for a time period $\Delta t$. A time step for the CDE solver of $0.5 \mathrm{~ms}$ for a $\rho$-grid of 100 points appears to be sufficient to avoid instabilities in the solver. Various integration times from $\Delta t=1 \mathrm{~ms}$ up to $\Delta t=100 \mathrm{~ms}$ were proven to be useful depending on the variability of the plasma and heating or current drive scenario. If for larger integration times $\Delta t$, e.g., the kinetic profiles or the current drive changes, the EC and NBI driven currents are recalculated as necessary. For a small $\Delta t=1-10 \mathrm{~ms}$ the change in the resulting current density compared to the initial one might be small for a large current diffusion time but results in similar equilibria as for a larger $\Delta t$ if the equilibrium is varying on a larger time scale only.

The second solver step of evaluating a new equilibrium at $t+\Delta t$ benefits from the fluxsurface averaged current distribution resulting from the CDE solver. The current distribution is augmenting the vector of measurements and the response matrix which calculates for a given equilibrium the ideal (noiseless, modelled) measurements and constraints. Typically 50 additional current constraints distributed equidistantly on the $\rho_{\text {pol-grid }}$ are provided to the equilibrium reconstruction.

The flux-surface averaged current distribution is assumed to have an absolute and 
COUPLING OF THE FLUX DIFFUSION EQUATION WITH THE EQUILIBRIUM RECONSTRUCTION AT ASD

relative uncertainty. An absolute uncertainty of $0.1 \mathrm{MA} / \mathrm{m}^{2}$ and a relative uncertainty of $10 \%$ is assumed which appears reasonable in view of the uncertainties involved, e.g., in the bootstrap current, ECCD and NBCD. The absolute uncertainty is necessary allowing for some flexibility in the edge (bootstrap) current distribution, which is well determined by the pressure constraints ${ }^{23}$. Furthermore, the absolute and relative uncertainties are necessary to avoid current holes in the reconstructed equilibrium which would be prone to numerical problems with the GSE solver. Additionally to the other measurements a least-squares criterion on predicted (CDE) and modelled (GSE) flux-surface averaged current distribution is applied. The equilibrium fits the flux-surface averaged current distribution according to the chosen tolerance. In case of non-circular plasmas external magnetic measurements do contain information about edge-localised moments of the current distribution ${ }^{9}$. Since magnetic measurements do not provide information about the current distribution in the plasma core, and, for the examples shown in the present work, no robust MSE or Faraday rotation data are available, the current distribution is determined only by the current data from the CDE solver. The resulting equilibrium at $t+\Delta t$ provides the new equilibrium for the next $\mathrm{CDE}$ solver step.

For any modelling code solving the CDE, e.g. TRANSP, the choice of the initial equilibrium might have a major effect on the temporal evolution of the equilibrium quantities. This initialisation problem is typically most problematic starting with a fully developed plasma with a large current diffusion time. Starting the equilibrium reconstruction at an early time point helps to avoid this initialisation problem due to the small current diffusion time accompanied with a large resistivity. Typically the first equilibrium at ASDEX Upgrade is calculated at 0.1-0.2 s after starting the plasma discharge where no auxiliary heating is applied and where the resistivity is rather large. Therefore, the current diffusion time is small. This results in a fast development of an equilibrium which does no longer depend on the initial equilibrium. Results will be shown below. 


\section{RESULTS}

First results of the coupling of the GSE and CDE solvers are presented for two examples: A counter-current ECCD plasma and the start-up phase of a plasma. The results of the countercurrent ECCD plasma will be compared with the results of a TRANSP analysis.

\section{III.A. Reversed-Shear Plasma}

The augmentation of the equilibrium reconstruction with the results of the CDE can significantly modify the current distribution compared to the standard equilibrium without current diffusion. Figure 3 shows results from the standard equilibrium without current
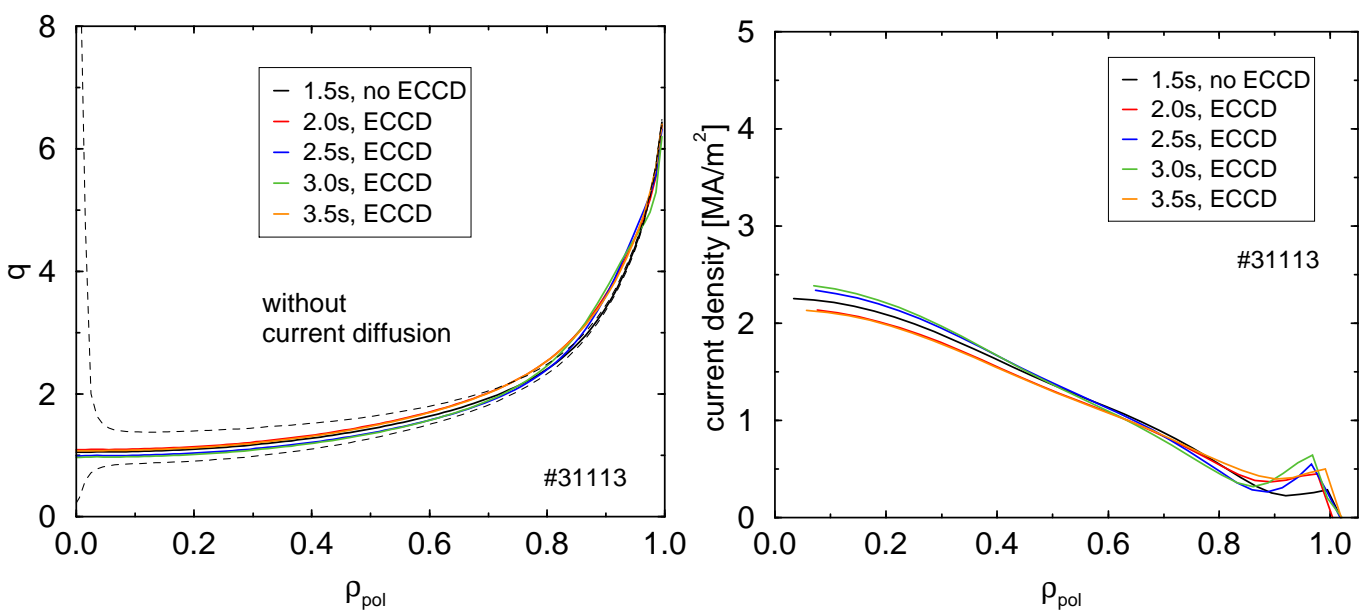

Fig. 3. Profiles of the safety factor $q$ and the flux-surface averaged toroidal current density without exploiting the CDE at various time points without and with ECCD.

diffusion. In the left panel the $q$-profiles for 5 time points (\#31113) are shown as a function of $\rho_{\mathrm{pol}}:=\sqrt{\left(\left(\psi-\psi_{\mathrm{ax}}\right) /\left(\psi_{\mathrm{sep}}-\psi_{\mathrm{ax}}\right)\right)}$ where $\psi_{\mathrm{ax}}\left(\psi_{\mathrm{sep}}\right)$ is the poloidal flux on the magnetic axis (separatrix), respectively. The first time point is shortly before 4 gyrotron launchers drive a significant amount of counter-current in the plasma center (see Figure 1). For the standard equilibrium reconstruction nearly no temporal variation of the $q$-profile is observed. This is also reflected in the flux-surface averaged toroidal current density profiles (right panel) 
before applying ECCD at $1.5 \mathrm{~s}$ and various time points with ECCD. The time interval is far beyond the current diffusion time. The lack of temporal variation in the current distribution must not be interpreted as a plasma phase with constant current distribution but is due to the lack of information about the current in the plasma core and the presence of (non-physical) smoothness constraints regularising the ill-posed equilibrium reconstruction. The effect of the smoothness constraints can also be seen in the uncertainty band of the $q$-profile (dashed lines) shown at $1.5 \mathrm{~s}$ only. Although the standard equilibrium in this example does not consider internal measurements of the current distribution, a relatively small uncertainty band arises which is merely due to the smoothness constraints. Weakening of the smoothness constraints results in an large increase (not shown) in the uncertainty band.

In contrast, coupling the GSE solver with the CDE shows a large effect on the evolution of the current distribution and, correspondingly, also on the $q$-profile. The left panel of Figure
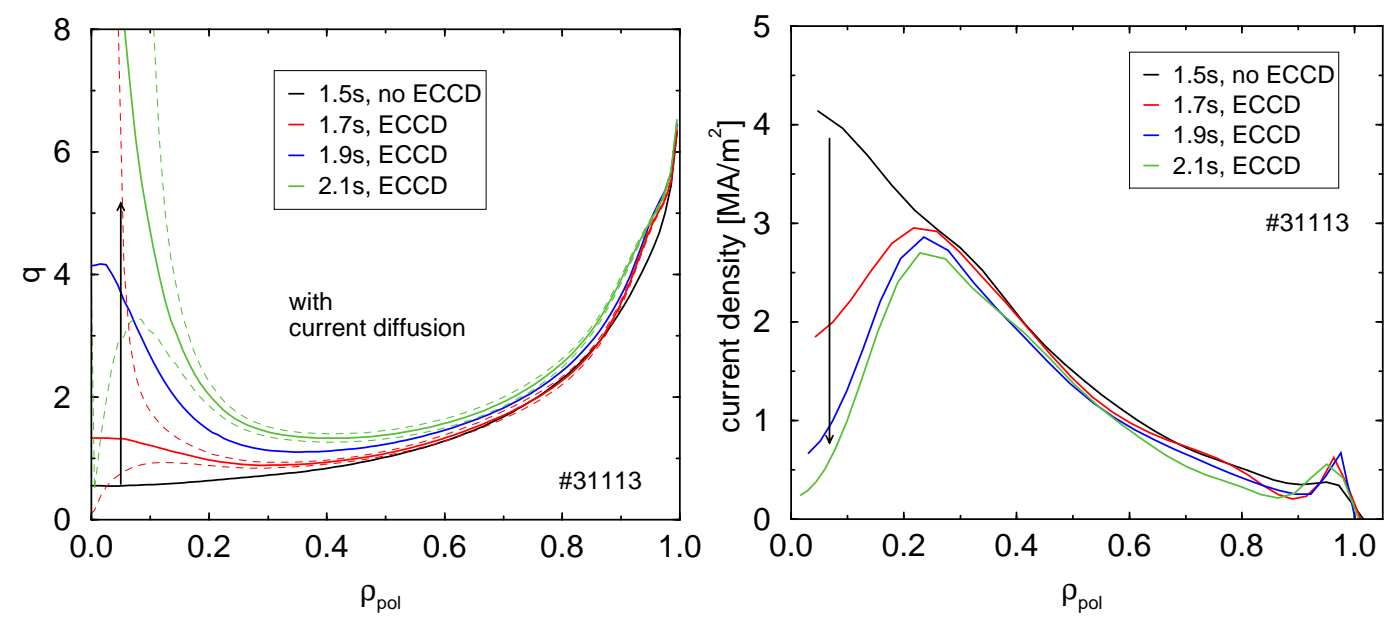

Fig. 4. Profiles of the safety factor $q$ and the flux-surface averaged toroidal current density using the CDE between subsequent equilibria.

4 shows the transition from a monotonically increasing $q$-profile without ECCD to a strongly reversed-shear $q$-profile with a large current depletion zone in the plasma center as strong counter current is driven. The uncertainty bands (dashed lines) represent the uncertainty of 
the current constraints. Owing to the additional information the smoothness constraints could and have to be reduced to allow for the necessary flexibility in the source profiles. For the same reason also the number of parameters (degrees of freedom) have to be increased from $22(11+11)$ to $26(13+13)$ spline knots for the source profiles $p^{\prime}$ and $F F^{\prime}$ with a larger knot density in the plasma core.
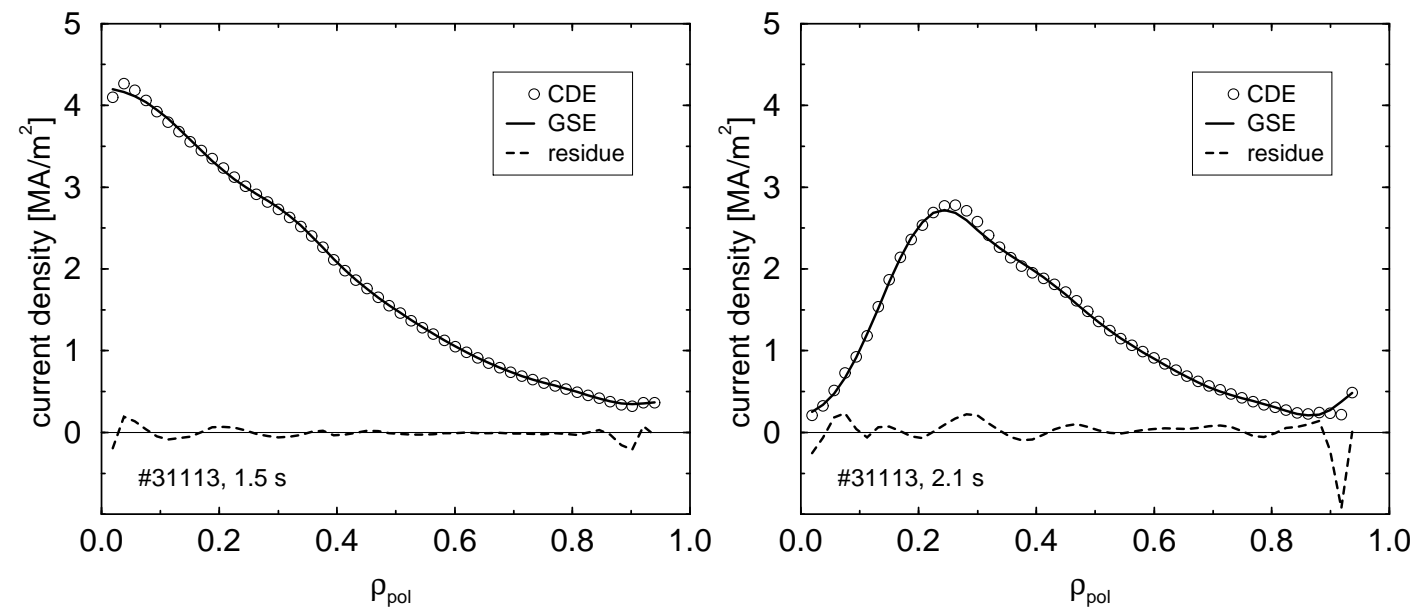

Fig. 5. Profiles of the predicted (CDE) and reconstructed (GSE) flux-surface averaged toroidal current density, and the residue of the two profiles weighted with the uncertainty of the predicted profile shortly before ECCD (1.5 s) (left panel) and after $0.6 \mathrm{~s}$ of counter-current $\operatorname{ECCD~(2.1~s)~(right~panel).~}$

Figure 5 depicts the flux-surface averaged toroidal current density predicted by the CDE solver (dots) and the corresponding current density resulting from the GSE solver constrained with the predicted profile (solid line). The left panel shows the profiles shortly before ECCD was switched on and the right panel after $0.6 \mathrm{~s}$ of counter-current ECCD $(2.1 \mathrm{~s})$. The dashed lines show the residues calculated from the difference of the CDE and GSE profiles divided by the uncertainty of the CDE profile. The GSE profile fits the constraint CDE profile very well. The good fit shows the lack of any further information about the current density in the plasma core as well as the sufficient flexibility in the parameterisation of the equilibrium. 

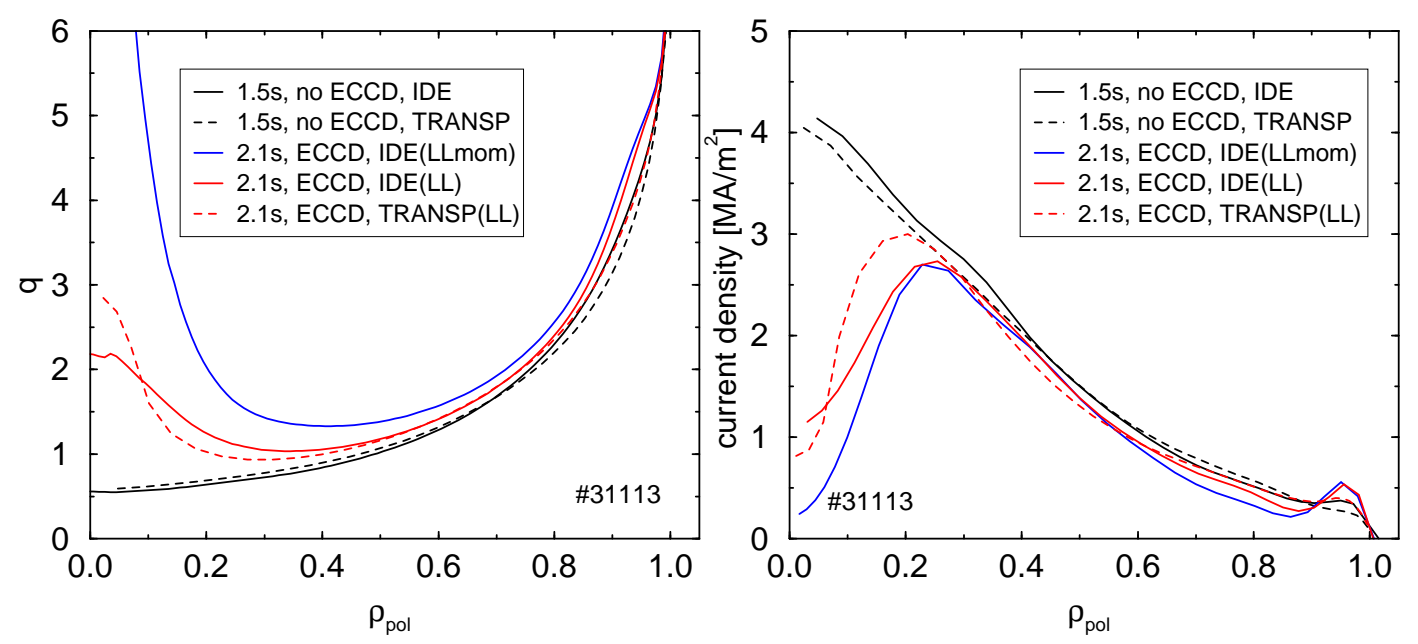

Fig. 6. Profiles of the safety factor $q$ and the flux-surface averaged toroidal current density shortly before ECCD (1.5 s) and after $0.6 \mathrm{~s}$ of counter-current ECCD (2.1 s) evaluated with the present (IDE) code (solid lines) and with TRANSP (dashed lines). LL (LLmom) refers to ECCD using the Lin-Liu (including momentum conservation) routine, respectively.

The strong evolution of the current density profile is confirmed by TRANSP calculations. Figure 6 shows the $q$-profiles (left panel) and the current density profiles for two time points shortly before ECCD $(1.5 \mathrm{~s})$ and after $0.6 \mathrm{~s}$ of counter-current ECCD $(2.1 \mathrm{~s})$. The solid lines depict the results of the present coupling scheme (IDE) and the dashed lines the results of TRANSP. IDE uses TORBEAM for ECCD whereas TRANSP uses the electron cyclotron heating and current drive code TORAY. Without ECCD at $1.5 \mathrm{~s}$ both codes show a monotonically increasing $q$-profile corresponding to a peaked current profile. After $0.6 \mathrm{~s}$ of counter-current ECCD, again, both codes show qualitatively similar results of current depletion in the plasma center and a reversed-shear $q$-profile. IDE was run with two different settings for TORBEAM evaluating ECCD. The red lines depict the $q$ - and current profile evaluated with the current drive routine as described in Lin-Liu ${ }^{24}$, whereas the blue lines show the profiles evaluated with the improved current drive routine including momentum conservation $^{25}$. The improved current drive was also used for the results shown in Figure 
1. As the driven current with the improved routine is, in our case, about $20 \%$ larger compared to the Lin-Liu routine, the depletion of the central current is faster and stronger. TRANSP(TORAY) uses the same Lin-Liu routine. Therefore, it is not surprising that the profiles of the IDE coupling scheme using the Lin-Liu routine and TRANSP(TORAY) agree also quantitatively very well. The difference of the two IDE results using Lin-Liu or the improved ECCD routine including momentum conservation shows the sensitivity of the results on the ECCD routine used.

The residual minor differences between the results are mainly due to the conservative assumption of the applied uncertainties of the current constraints and the weakened, but still present, smoothness constraints on the equilibrium source profiles. The proper choice of the smoothness constraints depends on the plasma scenario as a current depletion zone in the plasma centre necessitates a very relaxed smoothness condition.

\section{III.B. Start-Up Phase of a Plasma}

The second example shows the temporal evolution of the current distribution during the ohmic start-up phase of the plasma in the time interval $[0.15,0.9] \mathrm{s}$. Figure 7 depicts the development
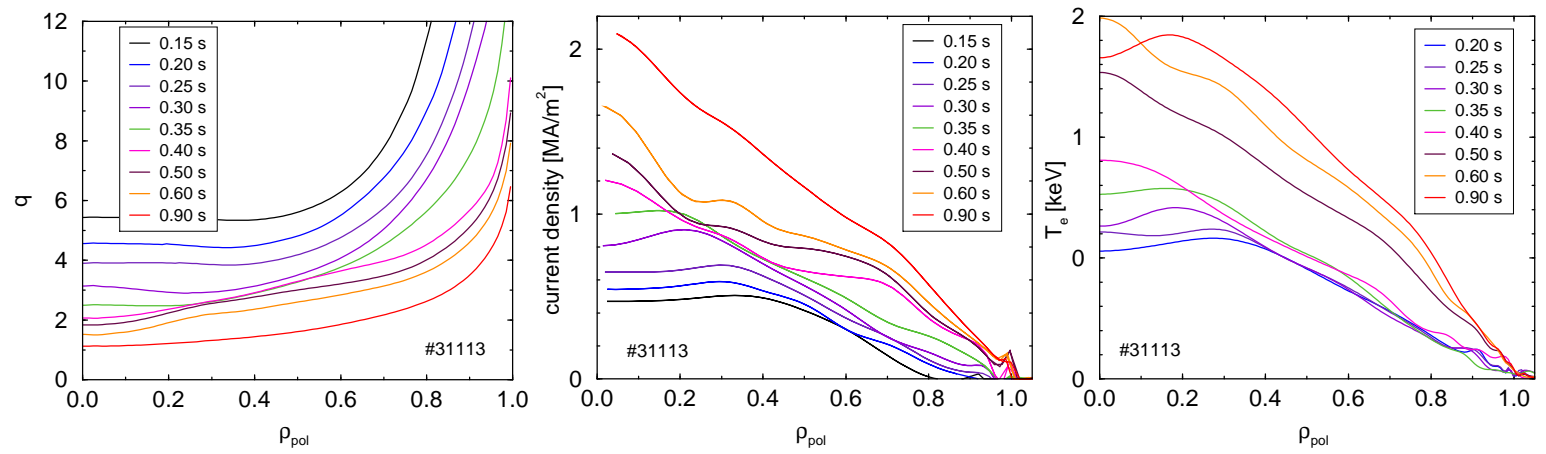

Fig. 7. Profiles of the safety factor $q$, the averaged toroidal current density, and the electron temperature for various time points in the plasma start-up phase.

of the $q$-profiles, the flux-surface averaged toroidal current profiles, and the corresponding 
COUPLING OF THE FLUX DIFFUSION EQUATION WITH THE EQUILIBRIUM RECONSTRUCTION AT ASD

electron temperature profiles $T_{\mathrm{e}} . T_{\mathrm{e}}$ at $0.1 \mathrm{~s}$ is assumed to be the same as for $0.15 \mathrm{~s}$ due to lack of data. It can be clearly seen that the current peak at $0.15 \mathrm{~s}$ at mid-radius is diffusing inwards resulting in an increased current peaking. The transition from a limiter plasma to a divertor plasma occurs at about $0.35 \mathrm{~s}$ determined from the time point where an X-point has formed without contact of the last closed flux surface to the wall. This transition can also be observed in an increase of the current density in $\rho_{\mathrm{pol}}=0.6-1.0$ corresponding to an increased temperature and, hence, conductivity in this area. Subsequently, a current density build-up occurs within this outer area. The $q$-profile shows an overall decrease up to about $0.35 \mathrm{~s}$ with a flat inner profile. Starting with the transition into a divertor plasma the shape of the $q$-profile changes. Due to the current increase the $q$-value reduces in the outer half of the plasma. With the subsequent current build-up and the inward diffusion the $q$-profile relaxes to the typical monotonic profile when the start-up phase is finished. At about $0.85 \mathrm{~s}$ the flat-top of the plasma current is reached. This detailed development of the current distribution cannot be obtained using the standard equilibrium as there is no information about the core-plasma current distribution provided.

Please note, that the results shown in Figure 7 are evaluated with an equilibrium evaluated every $5 \mathrm{~ms}$ where only each 10th equilibrium is shown in the figure. The plasma current increases approximately linearly from $300 \mathrm{kA}$ to $800 \mathrm{kA}$ within about $0.7 \mathrm{~s}$. This corresponds to an increase of the plasma current by about $3.6 \mathrm{kA}$ within one cycle time of $5 \mathrm{~ms}$. During this cycle time the boundary condition of the $\mathrm{CDE}$ given by the plasma current is kept constant. This approximation can be cured either by a shorter cycle time or by an adaptation of the boundary condition to the pre-programmed plasma current. Nevertheless, the effect can be neglected as long as the cycle time is small enough because the equilibrium is allowed to deviate within the uncertainty from the current constraint. The magnetic data ensure that after each cycle time the plasma current is at its correct value. 


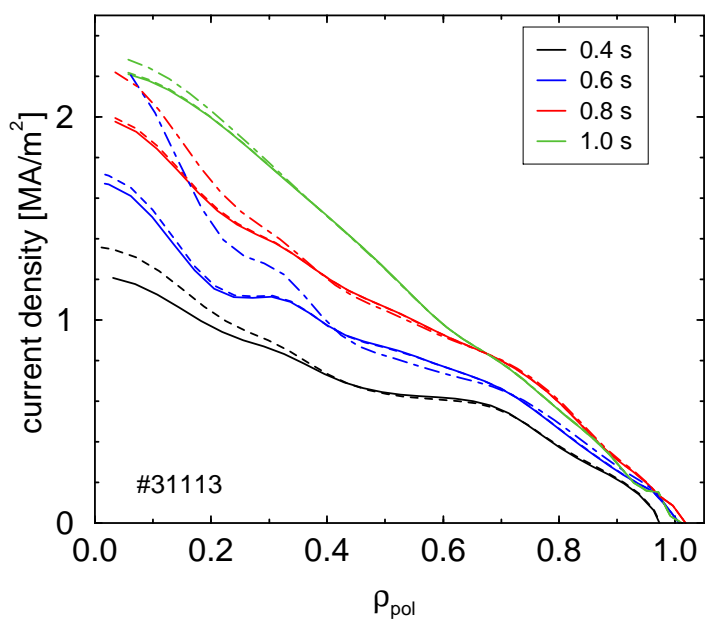

Fig. 8. Profiles of the flux-surface averaged toroidal current density at 4 time points for different starting times of the first equilibrium at $0.1 \mathrm{~s}$ (solid lines), $0.3 \mathrm{~s} \mathrm{(dashed),} \mathrm{and} 0.5 \mathrm{~s}$ (dot-dashed).

The benefit of a rather large resistivity at the beginning of the discharge for tackling the initialisation problem of reconstructing the equilibrium using the current diffusion is depicted in Figure 8. The current distributions are shown for 4 time points and 3 different starting times $t_{\mathrm{s}}$ of the equilibrium evaluation. The chosen starting times $t_{\mathrm{s}}$ are $0.1 \mathrm{~s}$ (solid lines), $0.3 \mathrm{~s}$ (dashed), and $0.5 \mathrm{~s}$ (dot-dashed, only 3 time points). The initial current distributions for the three starting times are not chosen to be identical. Instead, standard equilibrium reconstructions without current constraints are performed which result in different current distributions due to the different magnetic data.

The comparison of $t_{\mathrm{s}}=0.1 \mathrm{~s}$ (solid lines) with $t_{\mathrm{s}}=0.3 \mathrm{~s}$ (dashed lines) shows only a minor difference in the current distributions already at $0.4 \mathrm{~s}$. At $t>0.6 \mathrm{~s}$ the difference in the current distributions is barely noticeable. Starting at $t_{\mathrm{s}}=0.5 \mathrm{~s}$ results in a somewhat larger transient time due to the larger conductivity for $t>0.5 \mathrm{~s}$. Nevertheless, at $t=1.0 \mathrm{~s}$ the three current distributions coincide very well. Hence, starting the equilibrium reconstruction at an early time point, e.g. $t_{\mathrm{s}}<0.3 \mathrm{~s}$, helps to avoid the initialisation problem typically occurring 
COUPLING OF THE FLUX DIFFUSION EQUATION WITH THE EQUILIBRIUM RECONSTRUCTION AT ASD

when starting at fully developed plasmas with a large current diffusion time.

\section{SUMMARY AND OUTLOOK}

A scheme for the coupling of the current diffusion equation with the equilibrium reconstruction is proposed for the reconstruction of the equilibrium from measured data. This scheme is to overcome the ill-posedness of the equilibrium reconstruction in the plasma core in cases where no robust measurements of the current distribution exist. The method is dedicated for routine analysis shortly after the discharge where all diagnostic data are available but can also be applied to the coupling of the RAPTOR code with the real-time equilibrium calculation at ASDEX Upgrade. The scheme is to be interpreted as a link between the routinely used equilibrium codes running in offline mode without plasma modelling support and the highly sophisticated codes for plasma analysis and prediction.

The $\mathrm{CDE}$ is solved starting with the previous equilibrium up to the time point where the next equilibrium is to be evaluated. The set of measured data used for the reconstruction of the equilibrium is augmented with the flux-surface averaged toroidal current density resulting from the CDE solver. Results from the implementation of the CDE-GSE coupling within the IDE code package at ASDEX Upgrade and a comparison with TRANSP calculations were shown. The reversed-shear plasma with counter-current ECCD could be reconstructed without core plasma measurements. The temporal development of the current profiles agrees very well with TRANSP calculations if the same ECCD routine is used. The sensitivity of the results on the ECCD routine used is shown. An example of the start-up phase of the plasma shows the evolution of the current distribution. Although the present coupling of the CDE with the GSE is not as sophisticated as, e.g., the plasma modelling of Fable ${ }^{2}$ in the ramp-up phase, the evolution of the current distribution from the very beginning provides an important initialisation for the analysis of the fully developed plasma. 
ASDEX Upgrade is currently upgraded with an improved MSE diagnostic suite and Faraday rotation measurements which will allow to extend the validation of the improved reconstruction of the current distribution. Additionally, a sawtooth model will be included based on measured or predicted time points of the sawtooth occurrence ${ }^{26}$. To avoid the time consuming TRANSP calculations an approximation of the fast-ion pressure profile and the NBCD is under development.

\section{ACKNOWLEDGEMENT}

This work has been carried out within the framework of the EUROfusion Consortium and has received funding from the Euratom research and training programme 2014-2018 under grant agreement No 633053. The views and opinions expressed herein do not necessarily reflect those of the European Commission.

\section{REFERENCES}

\section{References}

[1] G. PEREVERZEV \& P. YUSHMANOV. ASTRA automated system for transport analysis in a tokamak. Tech. Rep. IPP 5/98, Max-Planck-Institut für Plasmaphysik, Garching (2002).

[2] E. FABLE, C. ANGIONI, F. CASSON, D. TOLD, A. IVANOV, F. JENKO, R. MCDERMOTT, S. Y. MEDVEDEV, G. PEREVERZEV, F. RYTER, W. TREUTTERER, E. VIEZZER, \& THE ASDEX UPGRADE TEAM. Novel free-boundary equilibrium and transport solver with theory-based models and its validation against ASDEX Upgrade current ramp scenarios. Plasma Phys. Control. Fusion, 55, 124028 (2013).

[3] E. FABLE, C. ANGIONI, A. IVANOV, K. LACKNER, O. MAJ, S. Y. MEDVEDEV, G. PAUTASSO, G. PEREVERZEV, W. TREUTTERER, \& THE ASDEX UPGRADE 
TEAM. Dynamical coupling between magnetic equilibrium and transport in tokamak scenario modelling, with application to current ramps. Plasma Phys. Control. Fusion, 55, 074007 (2013).

[4] R. BUDNY, M. BELL, H. BIGLARI, M. BITTER, C. BUSH, C. CHENG, E. FREDRICKSON, B. GREK, K. HILL, H. HSUAN, A. JANOS, D. JASSBY, D. JOHNSON, L. JOHNSON, B. LEBLANC, D. MCCUNE, D. MIKKELSEN, H. PARK, A. RAMSEY, S. SABBAGH, S. SCOTT, J. SCHIVELL, J. STRACHAN, B. STRATTON, E. SYNAKOWSKI, G. TAYLOR, M. ZARNSTORFF, \& S. ZWEBEN. Simulations of deuterium-tritium experiments in TFTR. Nucl. Fusion, 32, 429 (1992).

[5] F. FELICI, O. SAUTER, S. CODA, B. DUVAL, T. GOODMAN, J.-M. MORET, J. PALEY, \& THE TCV TEAM. Real-time physics-model-based simulation of the current density profile in tokamak plasmas. Nucl. Fusion, 51, 083052 (2011).

[6] J.-M. MORET, B. DUVAL, H. LE, S. CODA, F. FELICI, \& H. REIMERDES. Tokamak equilibrium reconstruction code LIUQE and its real time implementation. Fusion Engineering and Design, 91, 1 (2015).

[7] L. GIANNONE, R. FISCHER, P. MCCARTHY, T. ODSTRCIL, I. ZAMMUTO, A. BOCK, G. CONWAY, J. FUCHS, A. GUDE, V. IGOCHINE, A. KALLENBACH, K. LACKNER, M. MARASCHEK, C. RAPSON, Q. RUAN, K. SCHUHBECK, W. SUTTROP, L. WENZEL, \& ASDEX UPGRADE TEAM. Improvements for realtime magnetic equilibrium reconstruction on ASDEX Upgrade. Fusion Sci. Technol., ?, ? (2015). Submitted.

[8] R. FISCHER, J. HOBIRK, L. BARRERA, A. BOCK, A. BURCKHART, I. CLASSEN, M. DUNNE, J. FUCHS, L. GIANNONE, K. LACKNER, P. MCCARTHY, E. POLI, R. PREUSS, M. RAMPP, S. RATHGEBER, M. REICH, B. SIEGLIN, W. SUTTROP, E. WOLFRUM, \& ASDEX UPGRADE TEAM. Magnetic equilibrium reconstruction 
using geometric information from temperature measurements at ASDEX Upgrade. In V. NAULIN, C. ANGIONI, \& M. B. ET AL. (eds.), 40th EPS Conference on Plasma Physics, vol. 37D, page P2.139. European Physical Society, Geneva (2013).

[9] P. MCCARTHY. Identification of edge-localized moments of the current density profile in a tokamak equilibrium from external magnetic measurements. Plasma Phys. Control. Fusion, 54, 015010 (2012).

[10] R. FISCHER, C. FUCHS, B. KURZAN, W. SUTTROP, E. WOLFRUM, \& ASDEX UPGRADE TEAM. Integrated data analysis of profile diagnostics at ASDEX Upgrade. Fusion Sci. Technol., 58, 675 (2010).

[11] R. PREUSS, R. FISCHER, M. RAMPP, K. HALLATSCHEK, U. VON TOUSSAINT, L. GIANNONE, \& P. MCCARTHY. Parallel equilibrium algorithm for real-time control of tokamak plasmas. Tech. Rep. IPP R/47, Max-Planck-Institut für Plasmaphysik, Garching (2012).

[12] M. RAMPP, R. PREUSS, R. FISCHER, K. HALLATSCHEK, \& L. GIANNONE. A parallel Grad-Shafranov solver for real-time control of tokamak plasmas. Fusion Sci. Technol., 62(3), 409 (2012).

[13] M. RAMPP, R. PREUSS, R. FISCHER, \& THE ASDEX UPGRADE TEAM. GPEC, a real-time capable tokamak equilibrium code. Fusion Sci. Technol., ?, ? (2015). To be published.

[14] R. FISCHER, L. GIANNONE, K. LACKNER, R. MCDERMOTT, E. VIEZZER, H. ZEHRFELD, \& ASDEX UPGRADE TEAM. Effect of measured toroidal flows on tokamak equilibria. In 42th EPS Conference on Plasma Physics, page P1.117. European Physical Society, Geneva (2015).

[15] I. ZAMMUTO, L. GIANNONE, A. HOUBEN, A. HERRMANN, A. KALLENBACH, \& THE ASDEX UPGRADE TEAM. Long term project in ASDEX Upgrade: 
Implementation of ferritic steel as in vessel wall. Fusion Engineering and Design, $\mathbf{x x x ,}$ $\operatorname{xxx}(2015)$.

[16] S. RATHGEBER, R. FISCHER, S. FIETZ, J. HOBIRK, A. KALLENBACH, H. MEISTER, T. PÜTTERICH, F. RYTER, G. TARDINI, E.WOLFRUM, \& THE ASDEX UPGRADE TEAM. Estimation of profiles of the effective ion charge at ASDEX Upgrade with Integrated Data Analysis. Plasma Phys. Control. Fusion, 52, 095008 (2010).

[17] M. DUNNE, S. RATHGEBER, A. BURCKHART, R. FISCHER, L. GIANNONE, P. MCCARTHY, P. SCHNEIDER, E. WOLFRUM, \& THE ASDEX UPGRADE TEAM. Impact of Te and ne on edge current density profiles in ELM mitigated regimes on ASDEX Upgrade. Nucl. Fusion, 55, 013013 (2015).

[18] O. SAUTER, C. ANGIONI, \& Y. LIN-LIU. Neoclassical conductivity and bootstrap current formulas for general axisymmetric equilibria and arbitrary collisionality regime. Physics of Plasmas, 9, 2834 (1999).

[19] J. WESSON (ed.). Tokamaks. Clarendon Press, Oxford (2004).

[20] O. SAUTER, C. ANGIONI, \& Y. LIN-LIU. Erratum: "Neoclassical conductivity and bootstrap current formulas for general axisymmetric equilibria and arbitrary collisionality regime" [Phys. Plasmas 6, 2834 (1999)]. Physics of Plasmas, 9, 5140 (2002).

[21] E. POLI, A. PEETERS, \& G. PEREVERZEV. TORBEAM, a beam tracing code for electron cyclotron waves in tokamak plasmas. Computer Physics Communications, 136, 90 (2001).

[22] W. H. PRESS, S. A. TEUKOLSKY, W. T. VETterling, \& B. P. FlanNERY. Numerical Recipes. Cambridge University Press, Cambridge, 2nd edn. (1992). 
[23] M. DUNNE, P. MCCARTHY, E. WOLFRUM, R. FISCHER, L. GIANNONE, A. BURCKHART, \& THE ASDEX UPGRADE TEAM. Measurement of neoclassically predicted edge current density at ASDEX Upgrade. Nucl. Fusion, 52, 123014 (2012).

[24] Y. R. LIN-LIU, V. S. CHAN, \& R. PRATER. Electron cyclotron current drive efficiency in general tokamak geometry. Physics of Plasmas, 10, 4064 (2003).

[25] N. B. MARUSHCHENKO, C. D. BEIDLER, \& H. MAASSBERG. Current drive calculations with an advanced adjoint approach. Fusion Sci. Technol., 55(2), 180 (2009).

[26] F. PORCELLI, D. BOUCHER, \& M. ROSENBLUTH. Model for the sawtooth period and amplitude. Plasma Phys. Control. Fusion, 38, 2163 (21996). 